\title{
Theta burst stimulation for the acute treatment of major depressive disorder: A systematic review and meta-analysis
}

\author{
Jeffrey D. Voigt (1)', Andrew F. Leuchter ${ }^{2}$ and Linda L. Carpenter ${ }^{3}$
}

\begin{abstract}
Patients with major depressive disorder (MDD) may be refractory to or have contraindications that preclude treatment with antidepressant pharmacotherapies. Alternative therapies such as repetitive transcranial magnetic stimulation (rTMS) continue to evolve, and include theta burst stimulation (TBS), which has advantages over conventional rTMS. The aim of this study was to identify and meta-analyze efficacy data from all randomized controlled trials (RCTs) investigating TBS as a treatment for MDD. Published reports of RCTs (January 1, 2010 to October 23, 2020) were identified via systematic searches in computerized databases, followed by review of individual reports for inclusion. Inclusion criteria included primary diagnosis of $M D D \geq 1$ week duration of therapy with $\geq 10$ sessions, and treatment with any form of TBS. The Cochrane GRADE methodology and PRISMA criteria were used for evaluation of individual trials. Data from ten RCTs were included, representing 667 patients. Of these, 8 RCTs compared TBS to sham treatment and one compared TBS to standard rTMS (i.e., high frequency stimulation over left dorsolateral prefrontal cortex [HFL]). Quality of evidence assessment yielded high confidence in the finding of TBS being superior to sham on response measured by the Hamilton Depression Rating Scale (HRSD) (RR $=2.4 ; 95 \%$ Cl: 1.27 to 4.55; $\left.P=0.007 ; P^{2}=40 \%\right)$.

Comparison of HRSD response rates for TBS versus rTMS produced no statistically significant difference $(\mathrm{RR}=1.02 ; 95 \%$ Cl: 0.85 to $1.23 ; P=0.80 ; P^{2}=0 \%$ ). The incidence of adverse events between TBS and rTMS was not statistically different. The findings of a positive effect of TBS vs. sham, and noninferiority of TBS vs. standard HFL rTMS support the continued development of TBS to treat depression.
\end{abstract}

\section{Introduction}

Newer forms of repetitive transcranial magnetic stimulation (rTMS) have recently been developed, including theta burst stimulation (TBS) for the treatment of major depressive disorder (MDD). TBS sessions commonly last only 3-10 min, compared with conventional rTMS sessions (i.e., $10 \mathrm{~Hz}$ stimulation over the left hemisphere [HFL]), which can last up to $40 \mathrm{~min}$. Faster daily treatments with TBS may permit an increase in treatment capacity and the lowering of costs per session ${ }^{1}$.

\footnotetext{
Correspondence: Jeffrey D. Voigt (meddevconsultant@aol.com)

${ }^{1} 99$ Glenwood Road, Ridgewood, NJ, USA

'University of California Los Angeles (UCLA), Neuromodulation Division, Semel Institute for Neuroscience and Human Behavior, Los Angeles, USA Full list of author information is available at the end of the article
}

Furthermore, the brevity of daily TBS sessions makes undertaking a course of treatment more convenient and accessible for patients with demanding work schedules or other time commitments.

Several different TBS clinical protocols have shown efficacy for treating MDD: intermittent TBS (iTBS) applied to the left dorsolateral prefrontal cortex (DLPFC); continuous TBS (cTBS) applied to the right DLPFC; and consecutive bilateral cTBS/iTBS applied sequentially to the right and left DLPFC, respectively, in the same session. There have been systematic reviews and metaanalyses of randomized clinical trials (RCTs) in patients with MDD demonstrating that the antidepressant response with different forms of TBS is greater than the response to sham stimulation, with outcomes defined by 
the Hamilton Rating Scale for Depression (HRSD) ${ }^{2,3}$. However, direct comparisons between TBS and HFL rTMS treatment protocols on clinical outcomes (e.g., categorical response and remission rates) have not been made in a systematic review and meta-analytic manner to date. Additionally, newer RCTs have been published since 2017, so a comprehensive study of the currently available published data is important.

We performed an updated systematic review and metaanalysis of RCTs that evaluated TBS in MDD patients using devices with figure-eight TMS coils. The primary goal was to evaluate all published, peer-reviewed randomized controlled trials comparing TBS (unilateral or bilateral) to HFL rTMS or to sham therapy. We also examined relevant and widely accepted clinical outcomes/ endpoints for treating MDD.

\section{Methods}

A systematic search of electronic datasets was undertaken with the intention of including all languages of peer reviewed manuscripts and abstracts. Duplicate published studies were excluded except for cases where additional new data could be extracted from the original study.

The electronic datasets were searched on 10/23/20 and included: PubMed, EBSCO/CINAHL, and the Cochrane Central Register of Controlled Trials (CENTRAL). Searches were undertaken for the dates January 1, 2010 to October 23, 2020. Search terms used were as follows: (((((TMS) AND TBS) AND randomized AND control AND trial AND RCT) AND transcranial magnetic stimulation AND theta burst stimulation) AND major AND depressive) AND disorder. The references of identified RCTs and other systematic reviews and meta-analyses were also hand searched to identify any additional studies. Appendix 1 shows the results of the systematic search.

Peer-reviewed, published manuscripts and abstracts of RCTs were included as long as they met the inclusion criteria: patients $>18$ years of age, primary diagnosis of MDD, treatment with any form of TBS (intermittent, continuous, or derivations thereof including prolonged or combination protocols), comparison of TBS to HFL rTMS or TBS to sham, treatment $>1$ week duration; description of clinical endpoints and adverse events/ safety. Studies with both treatment naive (no prior MDD treatments) and treatment resistance prior to use of TBS were included. Where multiple publications of the same study existed, maximal data were extracted from these subsequent publications and included as part of the initial publication.

The primary outcome of interest was categorical response as measured by the clinician-rated Hamilton Rating Scale for Depression (HRSD) ( $\geq 50 \%$ reduction from baseline HRSD total score) at the end of the trial. Secondary outcomes of interest were adverse events,
HRSD percent change from baseline score, categorical remission (defined by HRSD 21-item final score <11), baseline-to-endpoint change on the self-report Beck Depression Inventory (BDI), and change on other (either self- or clinician-administered) anxiety or depression severity measures.

\section{Risk of bias assessment}

Two authors evaluated the risk of bias on randomization, treatment allocation, blinding (patient, treatment clinician, and treatment assessor), attrition, selective reporting, and "other" domains. Each domain was graded with regard to risk of bias as low, unclear, or high. Each domain outcome is described in the Cochrane risk of bias tool $^{4}$. A funnel plot to examine publication bias was also generated. A summary of the findings for included studies on risk of bias is included as Appendix 2, along with a list of, and reasons for excluded studies.

\section{Data synthesis and analysis}

Analysis was performed using the statistical software package contained in RevMan software from the Cochrane Collaboration ${ }^{4}$. Two or more studies which measured the same outcome were combined for metaanalysis. Based on assumptions that the true effect size varied amongst the included RCTs and that included trials would have substantial clinical and methodological diversity, a random effects model was employed ${ }^{5}$. For discrete outcomes, the statistical method used was Mantel-Haenszel with the risk-ratio (RR) for effect measures. For continuous outcomes, the inverse variance statistical method was used with standard mean difference (SMD) as the effect measure. Heterogeneity was measured in combined studies using the $I^{2}$ statistic to evaluate the consistency of findings. If heterogeneity was $>50 \%$, a sensitivity analysis was performed to identify the study causing the heterogeneity. If this occurred, the main results were reported with and without heterogeneity, and the sensitivity findings and potential reasons for the heterogeneity were described in the Discussion section. For studies which measured outcomes that were unique from others, the statistics as presented in the published report were included herein. Grading of Recommendations Assessment, Development and Evaluation (GRADE) profiler version 3.6.1 was used to assess the strength and the quality of evidence identified for clinical outcomes ${ }^{6-8}$. Lastly the PRISMA checklist was followed (Appendix 3$)^{9}$.

\section{Results}

\section{Literature search}

We screened 195 articles identified upon search from PubMed, EBSCO/CINAHL, and CENTRAL; 23 were retrieved for inclusion assessment. Ultimately 10 articles were included in the meta-analyses ${ }^{1,10-18}$. Of these, nine 


\section{PRISMA 2009 Flow Diagram}

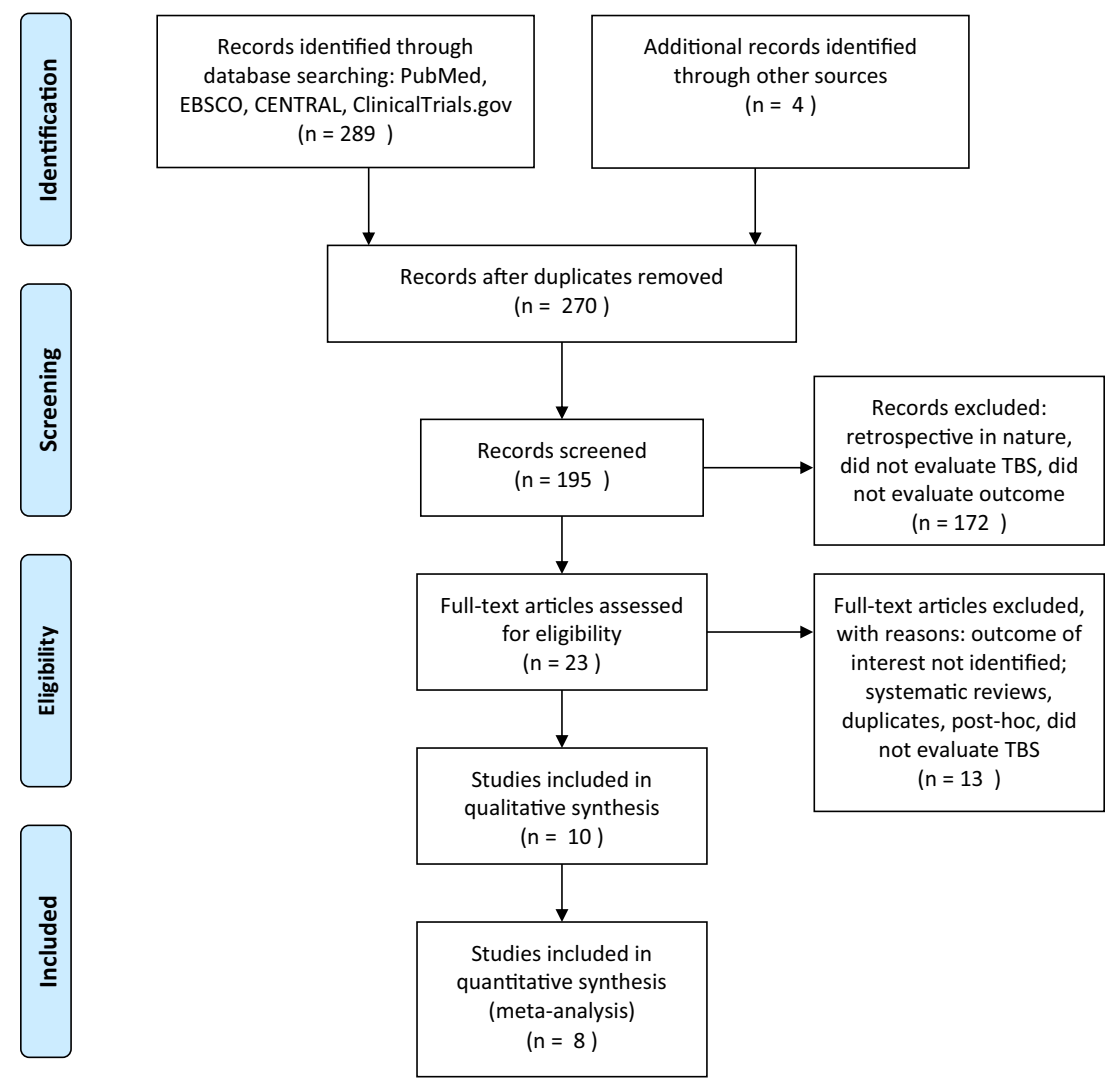

Fig. 1 PRISMA flow diagram. Systematic review process for identification of studies included in the analysis.

were single-center trials and one was a multicenter Canadian study ${ }^{1}$. Four studies took place in Germany ${ }^{12,16-18}$, two in Taiwan ${ }^{14,15}$, and one each in Australia $^{10}$, Belgium ${ }^{13}$, Canada $^{1}$, and Israel $^{11}$. There was a total of four studies that employed crossover designs, i.e., from TBS to sham and vice versa ${ }^{10-13}$. In these studies, clinical endpoints were examined prior to crossover to the other therapy. The flow chart in Fig. 1 depicts steps for the selection of studies for qualitative review and metaanalyses. Also, see Appendix 1 for more detail. Table 1 indicates which studies were used for meta-analysis in this study.

\section{Risk of bias assessment}

The randomization sequence (i.e., methodology used for random assignment) was identified as having low risk of bias in 4 out of 10 studies $^{1,13,14,17}$. Bias risk in blinding participants and personnel performing the therapy was low in 7 out of 10 studies $^{11-18}$; and bias risk in blinding outcome assessments was low in 8 out of 10 studies $^{1,11-15,17,18}$. In five studies there were high $(>10 \%)$ attrition rates among enrolled study participants ${ }^{1,10-12,17}$.
Three studies selectively reported information, including it in the results section but not identifying it as an endpoint in the methods section ${ }^{1,17,18}$. One study had evidence of potential conflict of interest, e.g. investigators or coauthors disclosed compensation as consultants to the company that funded the trial ${ }^{1}$. Figures 2 and 3 show a risk of bias graph and summary, respectively. Publication bias was also evaluated using a Funnel plot (Fig. 4), with the effect estimate (risk ratio) identified along the horizontal access and standard error identified along the vertical access. The resulting plot resembles a symmetrical inverted funnel thus reflecting an absence of publication bias on response outcome defined as HRSD reduction from baseline of $\geq 50 \%$.

\section{Clinical outcomes HRSD response}

Six studies evaluated categorical response $(\geq 50 \%$ reduction from baseline HRSD total score on the 17- or 21 - item scale; one study did not identify the number of HRSD items ${ }^{17}$ ) at the end of treatment weeks $1-6$ in TBS versus sham groups ${ }^{11,13-15,17,18}$. There was a significantly 


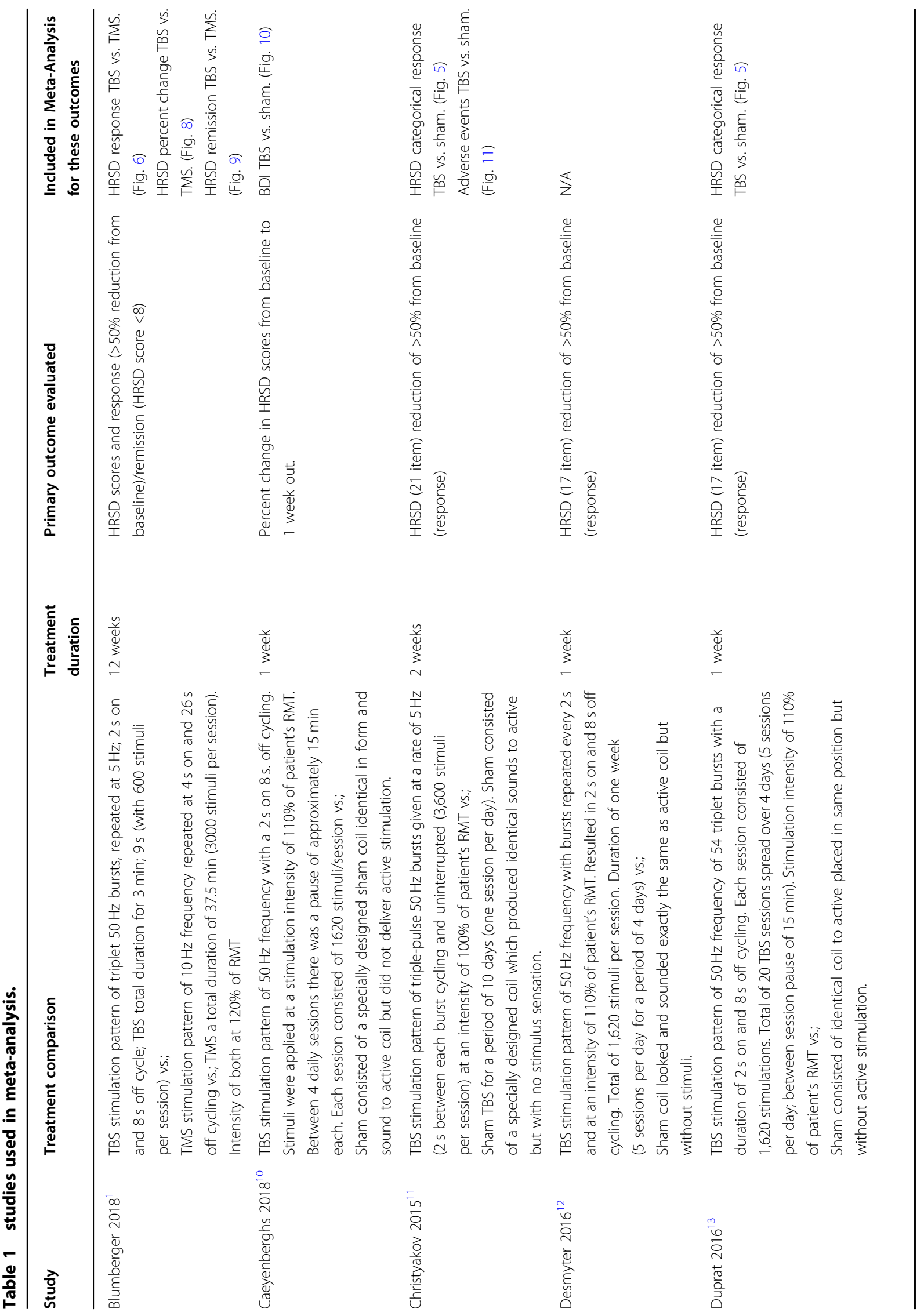




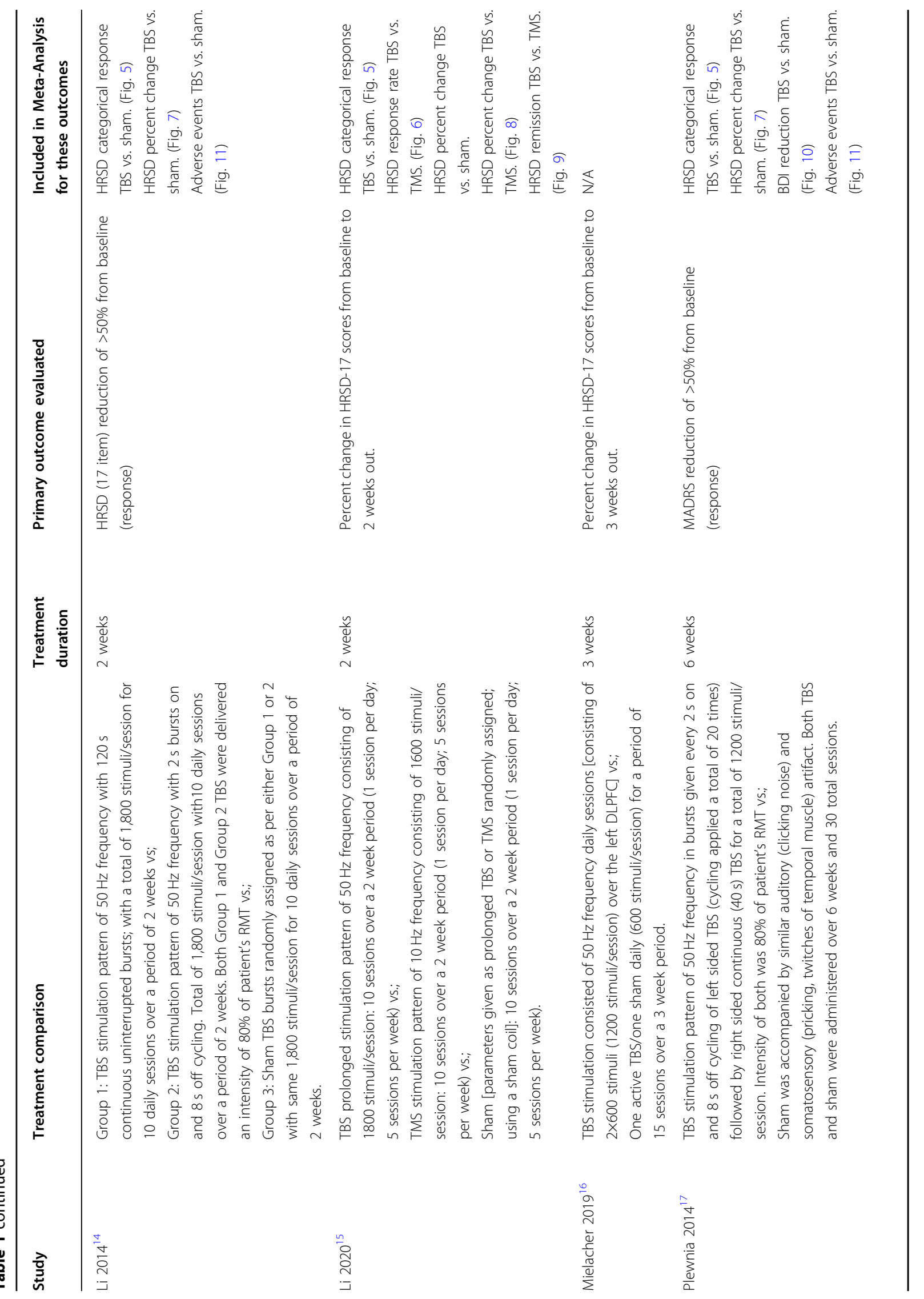


greater number of responders in the TBS than in the sham group ( $\mathrm{RR}=2.4 ; 95 \% \mathrm{CI}: 1.27$ to 4.55$) ; P=0.007$; $I^{2}=40 \%$ (Fig. 5). This outcome was also evaluated using the GRADE profile (Appendix 4). Due to the large effect TBS had on HRSD response, relative to sham, the quality of the evidence was graded as high, indicating high confidence in the estimate of the effect of the intervention. Desmyter et al. ${ }^{12}$ could not be included in the quantitative analysis as per Fig. 5 and in Appendix 4 based on how the data was presented in this crossover design study (sham data could not be extracted). However, it did demonstrate that $39 \%(18 / 46)$ therapy-resistant depressed patients showed $\geq 50 \%$ decrease in the HRSD scores. Comparison of HRSD response rates for those who got active TBS versus active rTMS (3 studies included in the analysis $^{1,15,18}$ ) produced no statistically significant difference between the 2 therapies when evaluated over a 2-12 week period; RR $=1.02 ; 95 \%$ CI: 0.85 to 1.23 ); $P=0.80 ; I^{2}=0 \%$ (Fig. 6).

\section{HRSD percent change}

Three studies examined HRSD percent change from baseline scores over $2-6$ weeks of treatment ${ }^{14,15,17}$. There was no statistical difference on this endpoint in the analysis of TBS versus sham in a meta-analysis including all three studies (SMD $=2.46 ; 95 \% \mathrm{CI}:-0.51$ to 5.42$) ; P=$ $0.10 ; I^{2}=97 \%$ (Fig. 7). Based on the high heterogeneity, a sensitivity analysis was performed with data from the $\mathrm{Li}$ et al., $2020^{15}$ trial excluded (based on the fact that there was no statistically significant difference in HRSD percent change with Li et al. 2020 and that there was a statistically significant difference in the other 2 studies favoring TBS), resulting in a statistically significant HRSD percent change from baseline favoring TBS (SMD $=0.6495 \% \mathrm{CI}$ : 0.13 to 1.16 ); $P=0.01 ; I^{2}=0 \%$. (See Discussion section below for elaboration of sensitivity findings for this outcome). When examining the HRSD percent change over a 2-6 week treatment period in analysis of studies comparing TBS vs. rTMS, again no statistically significant difference was found in the outcome (SMD $=2.92$; $95 \%$ CI: -3.05 to 8.90 ); $P=0.34 ; I^{2}=95 \%$ (Fig. 8).

\section{HRSD remission}

Categorical remission, defined as a final score $<11$ on the HRSD (21 item), was evaluated in one (TBS vs. sham) study $^{18}$. The authors reported no statistically significant difference in remission rates after 11 weeks ${ }^{18}$. Two studies defined remission by an endpoint HRSD (21 item) score $\leq 7$ and; there was no statistically significant difference between TBS and rTMS in the meta-analyses of this outcome evaluated at 2 and 12 weeks $^{1,15}$. However the results, favoring TBS, approached statistical significance $\left(\mathrm{RR}=1.39 ; 95 \% \mathrm{CI}: 0.98\right.$ to $1.98 ; P=0.06 ; I^{2}=$ 0\%) (Fig. 9). 


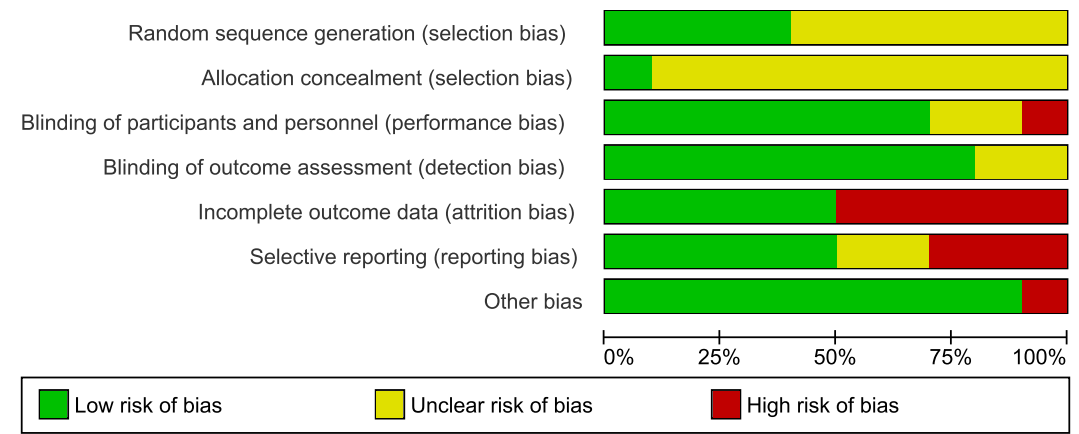

Fig. 2 Risk of bias graph. Aggregate evaluation of bias risks for studies included in the analysis.

\section{Beck depression inventory}

Absolute reduction in beck depression inventory (BDI) total score from baseline to post-treatment endpoint was evaluated in 2 studies comparing TBS vs. sham after a $1-6$ week duration of therapy ${ }^{10,17}$. There was no statistically significant difference between the TBS and sham groups (SMD $=-0.19$; 95\% $\mathrm{CI}:-2.13$ to 1.74$) ; \mathrm{P}=0.85$; $I^{2}=0 \%$ (Fig. 10).

\section{Other instruments and comparisons evaluated}

Outcomes defined by various other assessment instruments and summary metrics have also been used to compare the efficacy of HFL rTMS versus TBS. Results as reported in the individual published reports are summarized in Table 2, below.

\section{Adverse events}

All adverse event data were extrapolated as number of cases per event from the reports of RCTs comparing TBS and sham treatments ${ }^{11,14,17,18}$. The adverse events included both serious (e.g. suicide, hospitalization) and nonserious (e.g. headache, dizziness, nausea, pain) categories. There was no statistically significant difference between the TBS and sham groups when evaluating all adverse events: $\left(\mathrm{RR}=1.95 ; 95 \% \mathrm{CI}: 0.96-3.96 ; P=0.06 ; I^{2}=0 \%\right)$ (Fig. 11).

Pooled data from two TBS vs HFL rTMS RCTs also showed no statistically significant difference in adverse events $^{1,18}\left(\mathrm{RR}=0.99 ; 95 \% \mathrm{CI}: 0.88\right.$ to $1.12 ; P=0.89 ; I^{2}=$ $0 \%$ ) (Figure not shown). Self-rated intensity of pain during treatment, a non-serious event, was modestly greater with TBS than HFL rTMS as assessed by a verbal analogue 0 -to- 10 scale (mean score $3.8 \pm 2$ vs. $3.4 \pm 2 ; P=0.011$ ) in the one study which collected such data ${ }^{1}$.

Serious adverse events were reported in 4 of the TBS vs. sham RCTs ${ }^{11,14,17,18}$. There was no statistically significant difference between the groups ( $R R=5.00 ; 95 \% \mathrm{CI}: 0.26$ to 96.59); $P=0.29$ (Figure not shown). No serious adverse events were reported in two of the TBS vs. HFL rTMS $\mathrm{RCTs}^{1,18}$.

\section{Discussion}

In line with prior meta-analyses ${ }^{2,3,19}$, results of the current analysis demonstrated significantly higher response rates in patients with MDD following TBS than sham, as defined by a $\geq 50 \%$ reduction on HRSD. Based on the large treatment effect on this categorical response outcome measure as evaluated by GRADE, we found the strength and quality of the evidence for this finding to be high. There was consistency of findings across studies using the same instrument (HRSD) to define response, and consistency across outcomes derived from other instruments, all favoring TBS over sham. This likely bodes well for the robustness of our finding that TBS has a positive clinical effect on MDD.

Where high heterogeneity existed (i.e. in analysis of HRSD percent change comparing TBS versus sham), we found that eliminating one of the studies ( $\mathrm{Li}$ et al., 2020 ${ }^{15}$ ) resulted in a statistically significant difference favoring TBS. The difference in the eliminated $\mathrm{Li} 2020$ vs. the remaining included studies was that Li 2020 included patients who were trialed on only one pharmacologic medication and then remained medication free for a period of two weeks prior to starting stimulation sessions ${ }^{15}$. The remaining two studies both required resistance to at least two pharmacologic treatment trials and; subjects also remained on stable antidepressant medications prior to and during the course of study treatments ${ }^{14,17}$. Greater variation of clinical effect (measured as percent change in HRSD) may have arisen from inclusion of a population with relatively greater pharmacoresistance or more severe depressive illness in the two studies which were included in the meta-analysis after exclusion of $\mathrm{Li}$ et al. ${ }^{15}$. Thus this lower variation in the clinical effect found in Li 2020 (possibly due to a lower resistance to treatment effect with TBS) was different than the wider variation in effect found in both $\mathrm{Li} 2014^{14}$ and Plewnia 2014 ${ }^{17}$.

Updated data from our study extends the previously reported systematic review and meta-analytic literature comparing TBS to standard HFL rTMS by demonstrating 


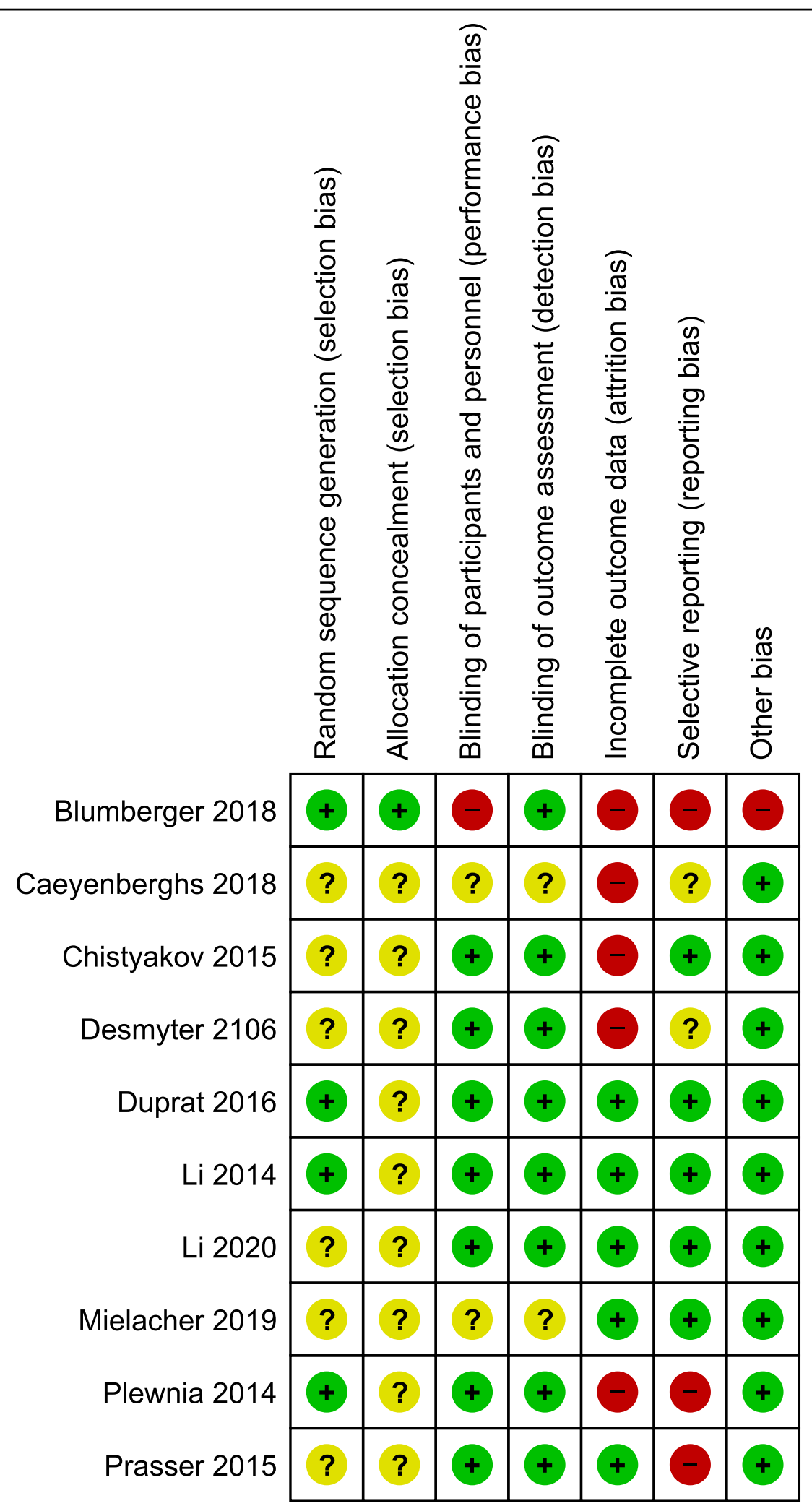

Fig. 3 Risk of bias summary. Specific biases as identified for each study included in the analysis. 


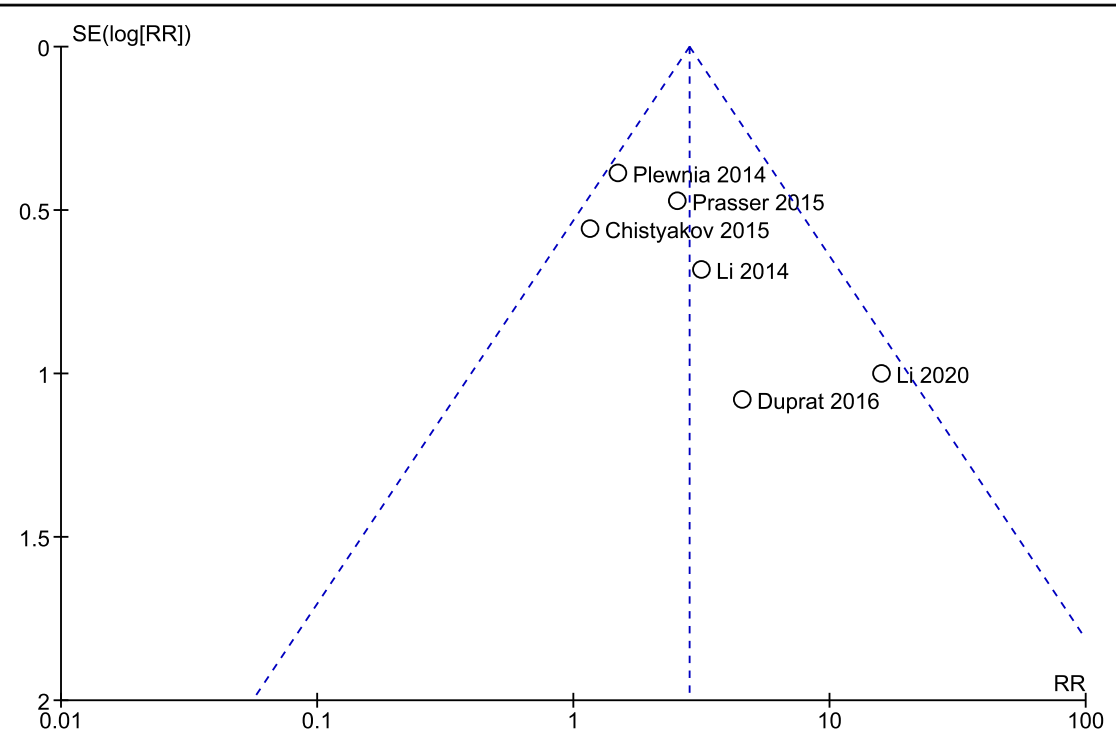

Fig. 4 Funnel plot examining publication bias. Intervention effect estimate from individual studies on HRSD response reduction from baseline measured against each study size.

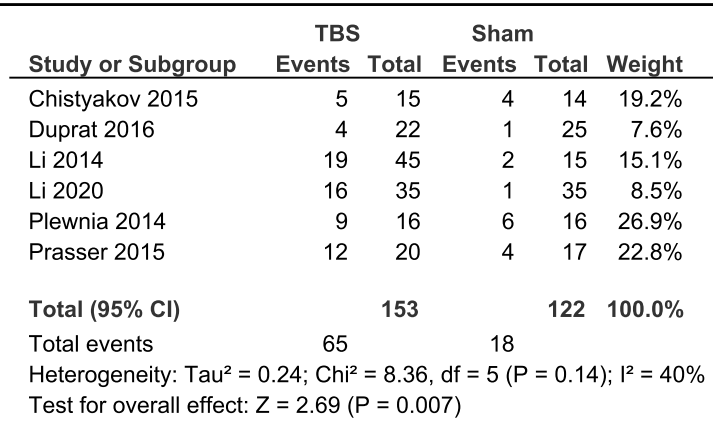

\section{Risk Ratio Risk Ratio}

Random, 95\% C

1.17 [0.39, 3.49]

$4.55[0.55,37.68]$

$3.17[0.83,12.03]$

$16.00[2.24,114.18]$

$1.50[0.70,3.23]$

$2.55[1.01,6.45]$

$2.40[1.27,4.55]$

M-H, Random, $95 \% \mathrm{Cl}$

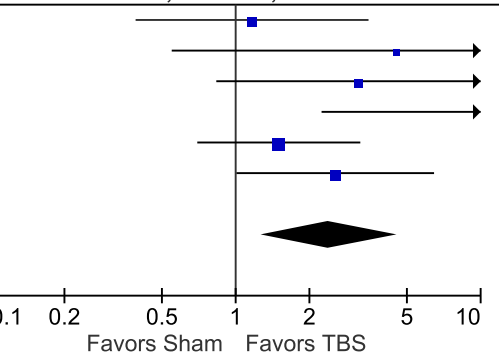

Fig. 5 HRSD score $\geq \mathbf{5 0} \%$ reduction (categorical response) from baseline TBS vs. sham. Forest plot displaying effect estimates and confidence interveals for both individual studies and overall meta-analysis. Each study is represented by a block at the point estimate of the intervention effect with a horizontal line extending on each side for the confidence interval. The area of the block indicates the weight assigned to that study in the meta-analysis.

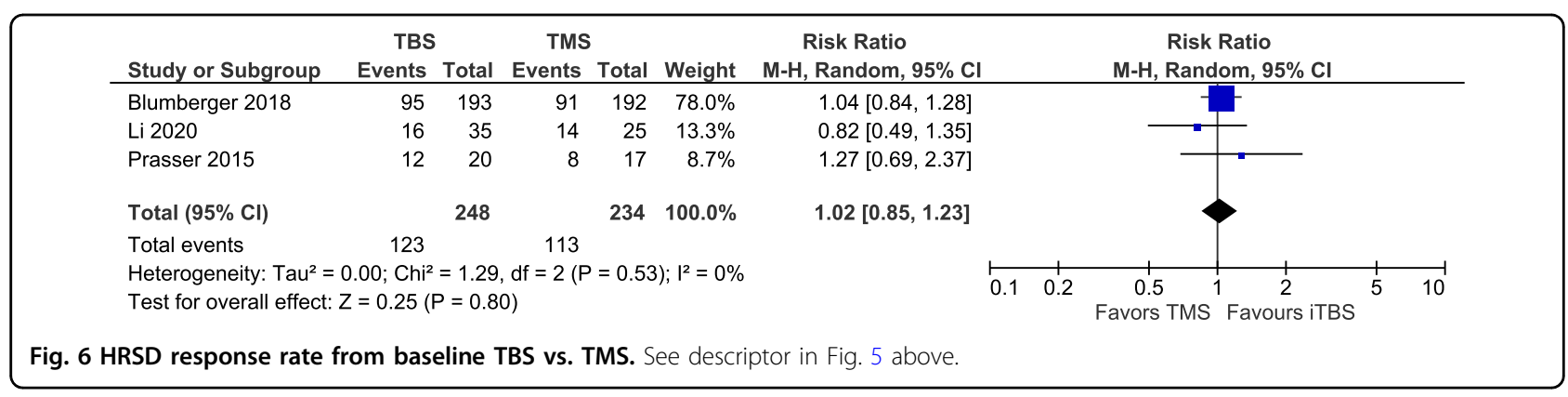

no statistically meaningful differences in response and/or remission outcomes on a number of validated clinician and self-report instruments, including HRSD, IDS-30, QIDS-30, MARDS, and BSI. While TBS efficacy was not statistically different to that of standard rTMS following a standard acute course, we note that this non-statistically significant difference also persisted for 8-12 weeks after treatment ${ }^{1,15,18}$. We also found no statistically significant difference in safety/adverse events between TBS and HFL rTMS therapies based on events reported in published 


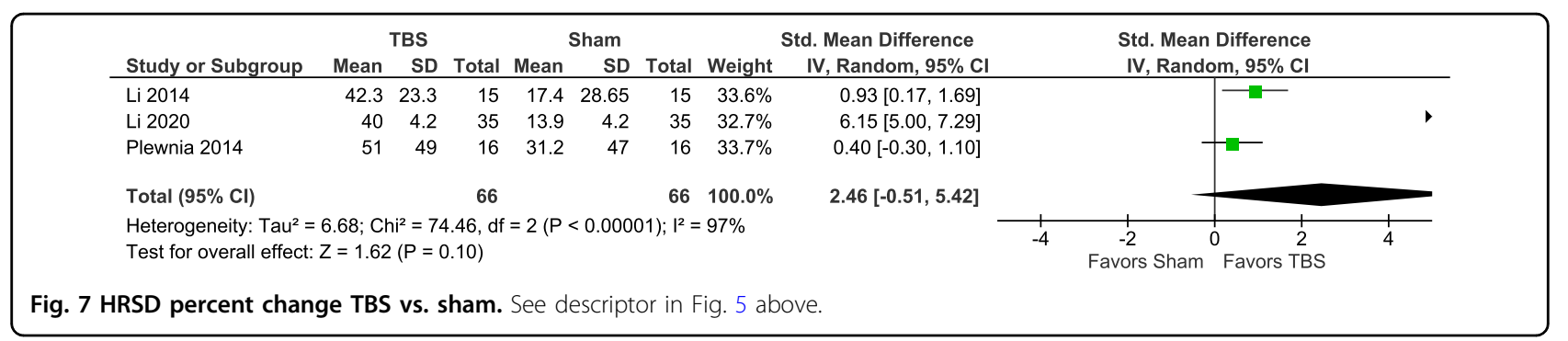

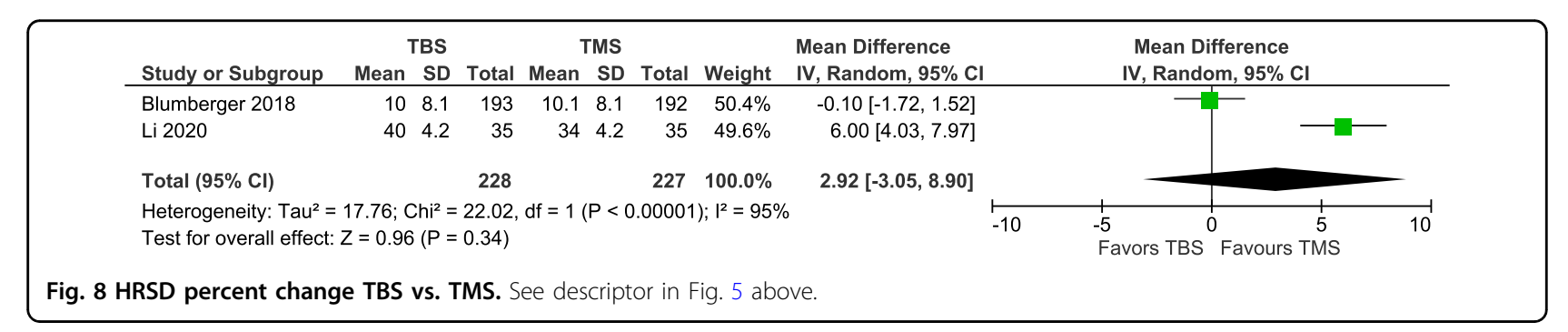

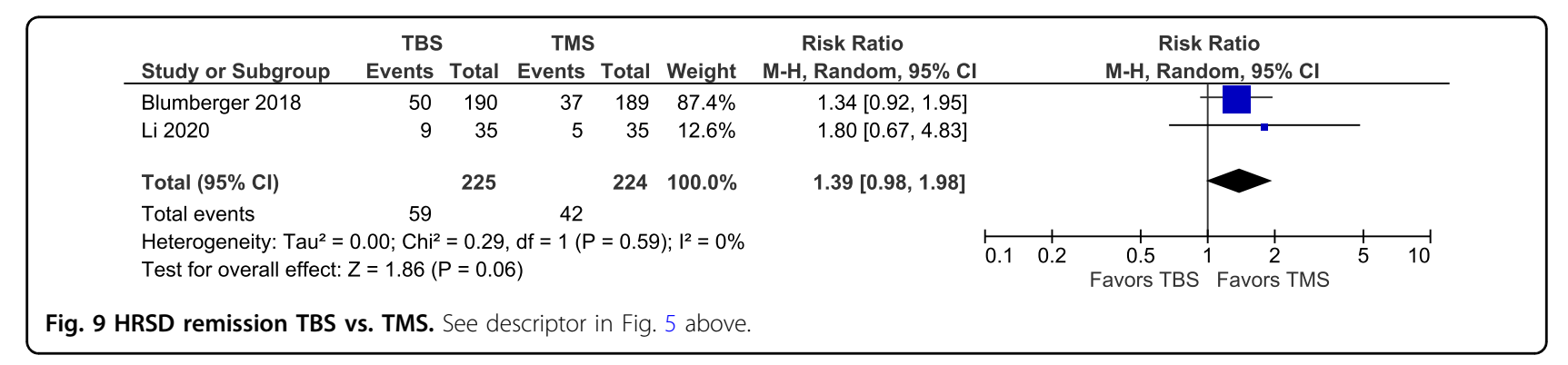

\begin{tabular}{|c|c|c|c|c|c|c|c|c|c|c|c|}
\hline Study or Subgroup & \multicolumn{3}{|c|}{ TBS } & \multicolumn{3}{|c|}{ Sham } & Weight & $\begin{array}{l}\text { Mean Difference } \\
\text { IV, Random, } 95 \% \mathrm{Cl}\end{array}$ & \multicolumn{2}{|r|}{$\begin{array}{c}\text { Mean Difference } \\
\text { IV, Random, } 95 \% \mathrm{Cl}\end{array}$} & \\
\hline Caeyenberghs 2018 & 2 & 13.15 & 21 & 5.5 & 17.18 & 31 & $5.5 \%$ & $-3.50[-11.76,4.76]$ & & & \\
\hline Plewnia 2014 & 9.5 & 3.49 & 16 & 9.5 & 2.08 & 16 & $94.5 \%$ & $0.00[-1.99,1.99]$ & & & \\
\hline \multirow{2}{*}{\multicolumn{12}{|c|}{$\begin{array}{l}\text { Total }(95 \% \mathrm{Cl}) \\
\text { Heterogeneity: } \mathrm{Tau}^{2}=0.00 ; \mathrm{Chi}^{2}=0.65, \mathrm{df}=1(\mathrm{P}=0.42) ; \mathrm{I}^{2}=0 \%\end{array}$}} \\
\hline & & & & & & & & & & & $\overrightarrow{10}$ \\
\hline
\end{tabular}

RCTs. In light of the notable advantage of TBS over standard HFL rTMS, i.e. significantly shorter duration of daily stimulation sessions, the noninferiority findings for TBS outcomes hold significant potential for shaping the current care standards for depressed patients. The 3 studies comparing TBS to rTMS included in the metaanalysis represent 482 patients and show a consistency of comparable outcomes between the 2 therapies. The findings, however, should be viewed with caution until more patients are reported on in similar type trials.

TBS appears to be well tolerated. We found the attrition rate (which incorporated the dropout rate) for TBS to be low in five studies, and even though it was somewhat higher $(\sim 10-12 \%)$ in the other five HFL rTMS studies, these rates are still significantly lower than the average $25 \%$ attrition reported for antidepressant medication trials ${ }^{20}$.

While this meta-analysis supports the preliminary efficacy of TBS treatment for MDD, there are questions regarding the optimal approach for administering TBS that remain unanswered. First, while response rates to TBS and other forms of rTMS treatment may be similar in the population of patients with MDD, it remains unclear whether these treatments are equivalent for individual patients. It is possible that there may be a differential response to TBS treatment based upon the degree of resistance to pharmacologic agents, clinical symptoms, or 
Table 2 Outcomes of other instruments assessed in single RCTs.

\begin{tabular}{llll}
\hline Study & Comparison & Instrument/outcome & Finding \\
\hline Blumberger $2018^{1}$ & rTMS vs. TBS & IDS-30; response $\geq 50 \%$ from baseline & TBS non-inferior to HFL rTMS \\
Blumberger $2018^{1}$ & rTMS vs. TBS & QIDS-SR; response $\geq 50 \%$ from baseline & TBS non-inferior to HFL rTMS \\
Blumberger $2018^{1}$ & rTMS vs. TBS & BSI-A; response $\geq 50 \%$ from baseline & TBS non-inferior to HFL rTMS \\
Blumberger $2018^{1}$ & rTMS vs. TBS & IDS-30; remission $<14$ & TBS non-inferior to HFL rTMS \\
Blumberger $2018^{1}$ & rTMS vs. TBS & QIDS-SR; remission $<6$ & TBS non-inferior to rTMS \\
Desmyter $2014^{12}$ & TBS vs. sham & BSI score & $P<0.01$ favoring TBS \\
Plewnia 2014 & TBS vs. sham & MARDS $\leq 50 \%$ from baseline; response & $P=0.048$ favoring TBS \\
Mielacher $2019^{16}$ & Once vs. twice daily TBS & HRSD percent reduction & $P=0.043$ favoring twice daily TBS \\
\hline
\end{tabular}

BSI Beck Scale for Suicide Ideation (Beck \& Steer 1991), BSI-A Brief Symptom Inventory-Anxiety subscale (BSI; Derogatis \& Melisaratos, 1983); IDS-3 Inventory for Depressive Symptomatology, 30-item (Rush et al. 1996); MADRS Montgomery-Åsberg, Depression Rating Scale (Montgomery \& Asberg 1979); QIDS-SR = 16 item Quick Inventory of Depressive Symptomatology (Self-Report version) (Rush et al. 2003).

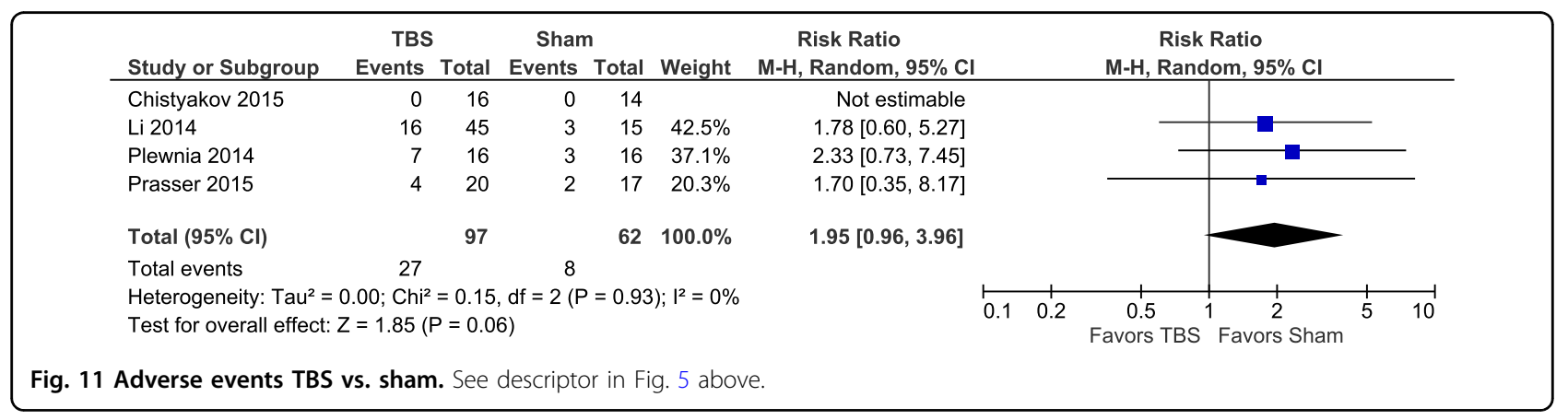

other sources of heterogeneity in the MDD population. For any individual patient, TBS or HFL rTMS may be superior. One recent report indicates that those patients who do not have an early response to HFL rTMS may show greater improvement following the addition of TBS priming (TBS-P) to their treatment regimen ${ }^{21}$. Future studies should examine this heterogeneity in response.

Second, it is important to note several factors that could have affected the findings including: considerable heterogeneity in the TBS protocols and patients reviewed herein, in terms of the number of pulses used; the number of sites stimulated; the fact that some trials allowed for continuation of medication regimens during the trial $^{1,11,14,17}$ and; number of failed trials $(1-3)$ prior to RCT enrollment. Li and colleagues ${ }^{14,15}$ have routinely applied 1800 pulses per session of TBS which may obtain superior clinical outcomes to studies employing 600-1200 pulses/session. As well, some of the RCTs applied stimulation at $80-100 \%$ intensity, relative to motor threshold, while others applied it at $110-120 \%$. There are a number of treatment protocols included in this systematic review and meta-analysis that include iTBS either alone or in combination with cTBS and prolonged TBS which are reflective of the attempts to identify an optimal treatment protocol for MDD. It is important to note that there may be complex non-linear effects from increasing the number of TBS pulses. The heterogeneity in these factors could not be controlled for in the present meta-analysis, but should be examined in further studies.

Third, one of the concerns in prior systematic reviews ${ }^{19}$ was a lack of follow-up assessment beyond the end of treatment. While the majority of studies did not have longterm follow-up assessments ${ }^{10,11,13-16}$ after courses of treatment lasting only 2-3 weeks, 2 studies had follow-up assessments and 8-12 weeks after the final stimulation $\operatorname{session}^{1,17}$. Blumberger et al. $^{1}$ identified a durability of response to TBS at 12 weeks (measured as a $>50 \%$ reduction from baseline on HRSD-17). At 8 weeks post-treatment, Prasser et al. ${ }^{17}$, found a non-significant trend towards a higher response rate $(>50 \%$ reduction from baseline on HRSD-21) with TBS compared to both rTMS and sham. Thus, there appears to be efficacy in regards to response rates with TBS lasting 2-3 months post-treatment.

Finally, there has been recent interest in the use of more than once-daily TBS treatments to speed treatment response. In one study, twice daily sessions of TBS significantly decreased depressive symptoms $53 \pm 23 \%$ vs. $36 \pm 35 \% \quad(P=0.043)$ when evaluated using percent 
reduction from baseline in the HRSD-17 instrument at the end of 3 weeks of treatment in comparison to once daily treatment (15 sessions $)^{16}$. While this was a small study of 36 patients comparing twice daily (active/active) vs. twice daily (active/sham), it demonstrated the likely need for protocols to evaluate optimization of the use of TBS in treating MDD. A recent open-label study used up to 10 daily sessions of iTBS in psychiatric inpatients and obtained robust response after only five days ${ }^{22}$.

The finding of positive effects of TBS on MDD confirm prior meta-analyses ${ }^{2,3,19}$, expand upon prior findings, and support the growing interest in application of TBS protocols for treating neuropsychiatric disorders such as MDD. This technique may present an opportunity for greater time- and cost-effective care with equivalent safety/adverse events profiles.

\section{Author details}

${ }^{1} 99$ Glenwood Road, Ridgewood, NJ, USA. ${ }^{2}$ University of California Los Angeles (UCLA), Neuromodulation Division, Semel Institute for Neuroscience and Human Behavior, Los Angeles, USA. ${ }^{3}$ Brown University Department of Psychiatry and Human Behavior, Butler Hospital TMS Clinic and Neuromodulation Research Facility, Los Angeles, USA

\section{Conflict of interest}

Dr. Carpenter reports research support from Neuronetics, Affect Neuro, Janssen, Nexstim, and Neosync. She has received income for consulting from Neuronetics, Affect Neuro, Nexstim, Janssen, Neurolief, Sage Therapeutics, Otsuka, and Sunovion. Dr. Leuchter discloses that within the past 36 months he has received research support from the National Institutes of Health, Department of Defense, CHDI Foundation, and NeuroSigma, Inc. He has served as a consultant to NeoSync, Inc., lonis Pharmaceuticals, Inc., and ElMindA. He is Chief Scientific Officer of Brain Biomarker Analytics LLC (BBA). Dr. Leuchter has equity interest in BBA. Jeff Voigt has in the past received consulting fees for reimbursement work performed for Magstim. However, he received no remuneration for this analysis nor; was Magstim aware of its existence.

\section{Publisher's note}

Springer Nature remains neutral with regard to jurisdictional claims in published maps and institutional affiliations.

Supplementary information The online version contains supplementary material available at https://doi.org/10.1038/s41398-021-01441-4.

Received: 20 January 2021 Revised: 3 May 2021 Accepted: 13 May 2021 Published online: 28 May 2021

\section{References}

1. Blumberger, D. M. et al. Effectiveness of theta burst versus high-frequency repetitive transcranial magnetic stimulation in patients with depression (THREE-D): a randomized non-inferiority trial. Lancet 391, 1683-1692 (2018).
2. Berlim, M. T., McGirr, A., dos Santos, N. R., Tremblay, S. \& Martins, R. Efficacy of theta burst stimulation (TBS) for major depression: an exploratory metaanalysis of randomized and sham-controlled trials. Jrl Psych. Res. 90, 109 (2017).

3. Brunoni, A. R. et al. Repetitive transcranial magnetic stimulation for the acute treatment of major depressive episodes. A systematic review with network meta-analysis. JAMA Psych. 74, 143-153 (2017).

4. Review Manager (Revman) [computer program]. Version 5.4. The Cochrane Collaboration (2020)

5. Riley, R. D., Higgins, H. P. \& Deeks, J. J. Interpretation of random effects metaanalyses. BMJ 342, d549 (2011).

6. Guyatt, G. H. et al. GRADE: an emerging consensus on rating quality of evidence and strength of recommendations. BMJ 336, 924-926 (2008).

7. Guyatt, G. H. et al. What is "quality of evidence" and why is it important to clinicians? BMJ 336, 995-998 (2008).

8. Schünemann H. J. et al. Chapter 12: Interpreting results and drawing conclusions. In: Higgins J. P. T., Green S. (editors), Cochrane Handbook for Systematic Reviews of Interventions Version 5.0.0 (updated February 2008). The Cochrane Collaboration, 2008. Available from www.cochrane-handbook.org.

9. Moher, D., Liberati, A., Tetzlaff, J. \& Altman, D. G. Preferred reporting items for systematic reviews and meta-analyses: the PRISMA statement. PLOS Med. $\mathbf{6}$, e1000097 (2009).

10. Caeyenberghes, K. et al. Accelerated intermittent theta burst stimulation in major depression induces decreases in modularity: a connectome analysis. Neuroscience 3, 157-172 (2018).

11. Christyakov, A. V. et al. Preliminary assessment of the therapeutic efficacy of continuous theta-burst magnetic stimulation (CTBS) in major depression: a double-blind sham-controlled trial. Jrl Affect Disor. 170, 225-229 (2015).

12. Desmyter, S. et al. Accelerated intermittent theta burst stimulation for suicide risk in therapy resistant depressed patients: a randomized, sham-controlled trial. Front Hum. Neurosci. 10, 480 (2016).

13. Duprat, R. et al. Accelerated intermittent theta burst stimulation treatment in medication-resistant major depression: a fast road to remission? Jrl Affect Disord. 200, 6-14 (2016).

14. Li, C.-T. et al. Efficacy of prefrontal theta-burst stimulation in refractory depression: a randomized sham controlled study. Brain 137, 2088-2098 (2014).

15. Li, C.-T. et al. Antidepressant efficacy of prolonged intermittent theta burst stimulation monotherapy for recurrent depression and comparison of methods for coil positioning: a randomized, double blind, sham-controlled trial. Biol. Psych. 87, 443-450 (2020).

16. Mielacher, C. et al. Once daily versus twice daily theta-burst stimulation in the treatment of major depression disorder. Brain stimulation 12, 397 (2019).

17. Plewnia, C. et al. Treatment of major depression with bilateral theta burst stimulation: a randomized controlled pilot trial. Jr. Affect Disord. 156, 219-223 (2014).

18. Prasser, J. et al. Bilateral prefrontal rTMS and theta burst TMS as an add-on treatment for depression: a randomized placebo controlled trial. World Jr Biol. Psych. 16, 57-65 (2015).

19. Somnez, A. I. et al. Accelerated TMS for depression: a systematic review and meta-analysis. Psych. Res. 273, 770-781 (2019).

20. Cipriani, A. et al. Comparative efficacy and acceptability of 12 new-generation antidepressants: a multiple-treatment meta-analysis. Lancet 373, 746-758 (2009).

21. Lee, J. C. et al. Strategies for augmentation of high-frequency left-sided repetitive transcranial magnetic stimulation treatment of major depressive disorder. Jrl. Affect Disord. 277, 964-969 (2020).

22. Cole, E. J. et al. Stanford accelerated intelligent neuromodulation therapy for treatment-resistant depression. Am. Jrl Psych. 177, 716-726 (2020). 